\title{
Genetic testing and common disorders in a public health framework
}

\section{Recommendations of the European Society of Human Genetics}

\author{
Carla G van $\mathrm{El}^{1}$ and Martina C Cornel ${ }^{\star, 1}$ on behalf of the ESHG Public and Professional Policy Committee
}

European Journal of Human Genetics (2011) 19, 377-381; doi:10.1038/ejhg.2010.176; published online 26 January 2011

Keywords: common disorders; genetic testing; predictive value; monogenic subtypes; public health; recommendations

\section{INTRODUCTION}

In recent years, the attention of the genomics and genetics research community has shifted toward understanding the basis of common disorders. The spectacular growth of genome-wide association studies has shed new light on the variants influencing risk factors. Understanding pathogenesis and etiology, and finding new ways to prevent and treat those diseases are major challenges. In the era of genomics, a promise of personalized prevention and drug treatment is presented, which many people meet with enthusiasm but which others call into question. The Public and Professional Policy Committee (PPPC) of the European Society of Human Genetics (ESHG), EuroGentest and the Institute for Prospective Technological Studies (IPTS) convened to discuss the relevance and possibilities of genetic testing for common disorders. Currently (in 2010), the genetics research community is skeptical about the possibilities of genetic susceptibility testing and screening contributing significantly to the improvement of the quality of health care. Meanwhile, some applications of very limited clinical utility have become available directly to consumers. Recently, the PPPC published critical recommendations on policy concerning DTC genetic testing (EJHG, 25 August 2010). When considering the potential of new genomic developments for a public health perspective, this Background Document takes the spectrum ranging from monogenic disorders on the one hand to common complex disorders on the other hand into account. It is argued that associations between genetic variants and disease risks of clinical relevance have been established, for instance for hereditary breast and ovarian cancer, colon cancer, diabetes mellitus (MODY subtypes), thrombosis, cardiovascular disorders, celiac disease and Alzheimer's disease. Although these examples relate to the monogenic subforms of common disease, they can nevertheless be used to reflect on the possibilities and relevant obstacles in using the new genetics in public health.

The deliberations, reflected in the final Background Document, have led to the below recommendations from the PPPC concerning the pitfalls and possibilities of genetic testing in common disorders.

A draft of both the Background Document and Recommendations has been distributed and posted on the web during the summer of 2009 to elicit further comments. The PPPC and the Board of the
ESHG approved the final version. This final text is considered to reflect the views of the European human genetics scientific and professional community.

\section{CONSIDERATIONS}

\section{Introduction}

After the focus on monogenic and chromosomal disorders in the second half of the twentieth century, in the last two decades research in genetics and genomics increasingly turned to common complex diseases. Common diseases are diseases frequently encountered in health care. We will use the term 'complex disease' to indicate diseases with variable etiology, including multifactorial etiology as well as monogenic subsets. When discussing 'susceptibility genes' in this document, we refer to genetic variants with low predictive value. By unravelling the contribution of monogenic subsets to the etiology of common disorders as well as the contributions of susceptibility genes to multifactorial etiology, including complex gene-gene and gene-environment interactions, this new research promises to give insights that might lead to refinement of diagnosis and more accurate prognosis, disease management and disease prevention in common complex disorders that are of major relevance for population health.

However, many of the claims that large-scale genetics and genomics research would contribute to better prognosis, therapy and prevention have not been substantiated yet, and might, in retrospect, have been too optimistic. More and more research findings on susceptibility genes do become available through genome-wide association studies. Whereas replication was difficult at first, various associations have increasingly been confirmed. There is an urgent need for the assessment of what is of clinical use vs what is not.

Translation of research findings to useful health-care applications appears to lag behind. Meanwhile, some applications that lack clinical utility have become available directly to the consumer. Difficulties with the translation of research findings need to be understood and addressed if genetics and genomics research is to fulfil its promises towards improving diagnosis, treatment and prevention. Currently (in 2010), the genetics research community is skeptical about the

\footnotetext{
${ }^{1}$ Department of Clinical Genetics/EMGO Institute for Health and Care Research, VU University Medical Center, Amsterdam, The Netherlands

*Correspondence: Professor M Cornel, Department of Clinical Genetics/EMGO Institute for Health and Care research, VU University Medical Center, Postbus 7057, BS7 D423, Amsterdam 1007 MB, Netherlands. Tel: +020 4448910 (direct) 4448914 (secr); Fax: +020 444 8181; E-mail: mc.cornel@vumc.nl
} 
possibilities of genetic susceptibility testing and screening contributing significantly to the improvement of the quality of health care. Meanwhile, the implementation in health care of genetic tests that are considered useful faces several thresholds.

Promises of improved diagnosis, therapy and prevention by genetics and genomics may in retrospect be interpreted as 'genohype': what was promised to funding agencies in order to obtain grants was also published in scientific journals and picked up by the media. In retrospect, several researchers have been aware of too high expectations.

To translate the research findings into appropriate clinical applications, such as genetic testing, several stages of assessment are needed. For the assessment of genetic testing and screening, several frameworks of criteria have been developed. Many of the parameters needed in these assessment frameworks are not available yet.

Furthermore, in Europe, the current regulatory framework does not cover an independent evaluation procedure for genetic tests before marketing to regular health care or directly to the consumer. Mechanisms for funding pre-market review and post-market surveillance are lacking.

Meanwhile, the general public is increasingly confronted with genetic tests, both for high-risk genes and for susceptibility, being offered on the internet without adequate regulation or independent sources of information and result interpretation.

At present, genetic testing for common disorders has been implemented in health care only in the case of some monogenic subforms of common disorders with highly penetrant mutations, such as the MODY subtypes of diabetes, breast and ovarian cancer, and colon cancer. Whatever can be learnt from these examples should not currently be extrapolated to the situation of susceptibility testing or screening on the basis of low-risk genes. The framework for such an endeavor needs to be explored.

These issues were discussed at a workshop in Seville, Spain, in October 2007, which was jointly organized by the PPPC of the ESHG, the EU-funded Network of Excellence, EuroGentest, and the IPTS. Experts and key actors, such as representatives of the Public Health Genomics Network, were present at the meeting. For the PPPC, the aim of the workshop was to produce statements and recommendations from the genetic health-care professional point of view. In June 2008, the preliminary documents were discussed at the ESHG meetings of the Board and PPPC in Barcelona. In order to further discuss some genetic epidemiological issues, a workshop was organized in September 2008 in Amsterdam. Suggestions were incorporated in both the background document and the recommendations. A draft of these statements and recommendations has been distributed and posted on the web to elicit comments during the summer of 2009. The PPPC and the Board of the ESHG approved the final version. This final text is expected to reflect the views of the human genetics scientific and professional community.

\section{Perspective}

In this document we will discuss genetic testing and common disorders from a health-care perspective. New possibilities for genetic testing confront health-care workers with the question whom to test and which test to use. The term 'common disorders' is used to denote that the disorders are relevant from a public health point of view because of their high frequency. Common disorders are, for instance, cardiovascular disease, stroke, diabetes, cancer, dementia and depression. For a health-care practitioner - unlike a geneticist or an epidemiologist - it is not clear, at the moment when a patient enters health care, whether the common disorder in this case is due to one gene with a high risk of serious disease, or due to a combination of several genes and several environmental factors. For disorders in which both etiological pathways exist, monogenic and multifactorial, when the patient enters health care the etiology can be thought of as 'complex'. Mendelian diseases can have a rather complex etiology too: one major gene, many modifying genes and a series of environmental factors. This is not what we mean by 'complex' in the present document.

Complex diseases are at present being redefined as a series of diseases with similar symptoms but variable etiology: a few genes, many genes, interactions between genes, one or a few environmental factors, many environmental factors, etc. The main difference between 'complex' and 'monogenic or Mendelian' is that in a Mendelian or monogenic disorder an alteration in one gene is a prerequisite for passing the threshold to develop the disorder, although the effect of the mutation could, in some cases, be modulated by genetic variants in other genes or epigenetic events caused by environment, life style, etc, while in complex disorders both whether one will develop the disease and the severity are modulated by the complex etiology. In Mendelian diseases one crosses the threshold to disease due to one gene; in complex diseases different factors are needed for crossing the threshold. Between these two extremes one will find a series of combinations, for example, BRCA, where one gene will bring you close to the threshold but other genes and/or environmental factors will push you over it. Mendelian diseases and subtypes can be of major importance as an example for common complex diseases, both to generate insights into etiological pathways and in avenues for the potential implementation of specific programmes in health care.

\section{Definition of genetic testing vs screening}

There are several valid definitions of genetic testing and screening available. For the purpose of this document we will use genetic (susceptibility) 'testing' in a broad sense, while we use genetic (susceptibility) 'screening' for a systematic, proactive offer to members of a certain group of individuals. Screening may be a well-organized public health program, usually aiming at a low-risk population. 'Genetic' testing will be defined as the analysis of DNA or biomarkers for the evaluation of one or more genetic risk factors for a particular disease or disease group.

\section{Evidence for causality}

For monogenic subtypes of common complex diseases, the relation between genetic alterations in genes conferring a high disease risk and the disease is well-established - for instance, in the case of breast and ovarian cancer caused by BRCA1 and BRCA2 mutations, familial adenomatous polyposis (FAP), hereditary non-polyposis colorectal cancer (HNPCC), MODY subtypes of diabetes and familial hypercholesterolemia (FH). For monogenic subtypes the predictive value is high, although the penetrance is not complete. Furthermore, there is a causal relation between the genetic alteration and the disease.

The number of genome-wide association studies revealing association between genetic variants and common complex disorders is rising rapidly. Although evidence is accumulating and several associations have been confirmed, still much work is needed on the replication of associations between genetic variants and diseases. Especially, comparison of the results of association studies between populations is a challenging issue. Furthermore, gene-gene interactions as well as interactions between genetic and environmental factors merit further study to better understand causal pathways and their potential clinical use.

For many associations identified recently however, the predictive value is low; the proportion of people with the variant associated 
with increased risk that will develop the disorder is not much higher than the population risk, and the variant does not represent a causal variant.

\section{Translation of research findings}

As yet, promises about health-care applications on the basis of recent research findings in genetics or genomics research have been overstated. There is hardly any effect on improving diagnosis and prognosis, treatment and prevention, such as lifestyle interventions or effective therapies concerning common complex disorders on the basis of these newly established associations. Translation of research findings on genes with low predictive value into health-care practices - be it public health, primary or specialized health care - is problematic. The main criterion to assess whether (a combination of) tests could improve health care is whether it is a good predictor at the population level. Furthermore, effective interventions need to be available. Most genetic variants may only alter the disease susceptibility risk by a factor of 1.1-1.6, and usually a large number of genetic variants will have a bearing on the risk, of which only a minority will be known or included in test panels. In most cases of polymorphic variants, the predictive value would be too small for any intervention to be appropriate, and it is disputed whether a combination of risk variants could confer a sufficiently high relative risk to support the recommendation of testing or screening in case risk-reducing interventions become available.

In the near future, the use of multiple genetic variants for, for instance, colorectal and breast cancer might allow individuals at substantially increased risk to be identified. These gene variants for which the predictive value is high enough to be useful for clinical application are monogenic subtypes. Combining multiple SNPs will probably not provide adequate prediction for targeted interventions. At present, for certain disorders family history is almost always a more useful way to stratify the risk.

In the case of moderate-risk genes, there has been some success in improving diagnosis - for instance, in the case of determining HLA subtypes to confirm the diagnoses of Bechterew's disease and celiac disease. In case of several monogenic subtypes of common disorders diagnostic tests are in widespread use, as shown by the earliermentioned examples of breast and ovarian cancer related to BRCA1 and BRCA2 mutations, FAP, HNPCC, MODY and FH.

\section{Assessment}

In the past decades the criteria for assessing genetic tests and genetic screening programs have been elaborated. The comprehensive ACCE framework from the Centers for Disease Control and Prevention (USA) has set a contemporary standard for assessing tests. Several groups have further adapted this framework, which evaluates not only the test characteristics but also the medical setting in which the test is applied, as well as the legal, social and ethical context of the medical application of single-gene tests. According to the ACCE framework, a test should first of all have a good analytic validity: this is a measure of the technical accuracy of the test and defines the test's ability to measure accurately and reliably what it is aiming to identify. Clinical validity defines a test's ability to detect or predict the disorder of interest. Clinical utility refers to the likelihood that the test will lead to improved health conditions when introduced to clinical use. Information on analytic validity alone is insufficient to assess the usefulness and performance of a test in medical services.

Assessment frames such as the ACCE model have been developed for single-gene disorder tests. Would these principles be applied to the assessment of genetic testing in common complex disorders, information about many aspects would not be available yet. To evaluate clinical utility in a specific setting, test characteristics (sensitivity, specificity in a diagnostic or screening context), knowledge of the disease (prevalence, severity) and intervention results (reduction of morbidity and mortality; psychological consequences) are needed. In addition, in many cases information is lacking regarding the relation between the tested genetic variant and other genes, gene variants or combinations of gene variants relevant for a certain disorder, and the safety and usefulness of interventions such as medication or lifestyle changes. Therefore, the PPPC statement from 2003, 'It will take many years to be sure that the identification of groups at risk for common diseases, or for specific drug therapies and consequent interventions, is beneficial', is still valid for many health-care settings.

In the absence of sufficient information on clinical validity and clinical utility, introduction of genetic tests in common complex disorders is often premature. In the near future, in a certain healthcare setting, genetic tests in common complex disorders may be introduced for tests and settings where clinical utility is likely, to gain additional information concerning diagnosis, prognosis and disease management. However, this introduction should be carefully monitored in order to obtain additional information regarding the usefulness and performance of the test in that specific setting.

\section{Priorities}

When considering implementation in health care, priority should be given to genetic tests for common complex diseases of proven clinical utility and cost effectiveness. Taking a family history can, for some disorders, be an adequate initial source of risk differentiation, enhancing both the efficiency and cost effectiveness of further testing.

In case of monogenic subtypes of common disorders, family history may be followed by cascade screening of family members at risk. This would provide ready opportunities to health gain in the case of, for instance, breast and ovarian cancer related to BRCA1 and BRCA2 mutations, FAP, HNPCC, MODY subtypes of diabetes and FH. Examples of testing for monogenic subtypes of common disorders have been implemented in health care because of higher predictive value as well as the availability of interventions.

In some cases, applications related to the specification of tumor diagnoses or the choice of therapy are feasible, such as in case of the systematic testing of mutations in breast cancer tumor tissue, or pharmacogenetic testing in case of certain adverse drug reactions or drug dose determination.

At present, genetic testing for common disorders in other cases is often not considered useful.

\section{Direct-to-consumer tests}

Increasingly, genetic tests for common disorders can be obtained via the internet or over the counter as a direct-to-consumer test, without thorough assessment of the clinical validity and utility. As information on analytic validity alone is insufficient to assess the usefulness and performance of a test, this information should not be considered as sufficient pre-market evaluation.

The premature introduction of commercial genetic tests may have some serious disadvantages. The test results may be confusing or raise concern, may cause false distress or reassurance, and people may need extra information from health professionals to explain the test results and possible consequences or courses of action. This would mean the extra use of scarce health-care resources, or the test may simply be a waste of money. Premature introduction of genetic susceptibility tests may therefore also seriously undermine public trust in genetic testing for medical purposes. 
Often, interpretation of test results in relation to other health and lifestyle indicators of an individual is necessary, which may include relevant family history and other risk factors regarding a certain disorder. To adequately interpret genetic and other risk information, we recommend that advice from sufficiently qualified health-care professionals be available when direct-to-consumer genetic tests for common disorders are offered. Where applicable, they should be able to provide genetic counselling.

Special attention is necessary regarding advertising for direct-toconsumer tests to ensure adequate information is given and truthful claims are made about the test and possible interventions. To evaluate whether the claims are truthful and to evaluate the performance of tests (esp. clinical utility), involvement of health authorities is necessary.

\section{Regulation}

Regulation is necessary to improve the assessment procedures of genetic tests for common disorders, either to be used in health care or to be offered as direct-to-consumer tests on the internet.

In European regulation, many genetic tests fall under the heading of in vitro diagnostics (IVD). Currently, in the IVD Directive, these tests have often been categorized as low-risk products and are therefore not subject to independent evaluation before coming to market. Thus, clinical validity and utility may not be assessed.

The goal of the process of pre-market review is to ensure truth-inlabelling and truthful promotion of in vitro diagnostic devices. Mechanisms for post-marketing evaluation are not in place yet. It is recommended that an internationally recognized regulation for mandatory pre-market review for genetic tests be established, including one for common disorders. It would be desirable to develop a postmarketing evaluation procedure.

Member countries differ in their interpretation of the IVD Directive. New guidance should be consistent with the OECD guidelines for Quality Assurance in Molecular Genetic Testing and the Council of Europe's Additional Protocol to the Convention on Human Rights and Biomedicine, concerning Genetic Testing for Health Purposes: http://conventions.coe.int./treaty/EN/Treaties/Html/203.htm

\section{Harmonization}

Within the European Union, initiatives by various actors should lead to a combined effort to harmonize regulation concerning genetic testing and screening, including genetic testing for common disorders between the member states. Harmonization would be facilitated by ensuring consistency with the Council of Europe's protocol.

Harmonization between the European Union and the United States regarding the assessment of genetic tests for common disorders is desirable.

However, harmonization should not be restricted to regulation per se. Actors from various fields of interest should reach common ground, for instance, regarding professional protocols, standards for referral, and codes of practice applicable in health care and public health.

\section{Commercial valorization and responsible entrepreneurship}

At present, funding for a pre-market assessment procedure is lacking. On the other hand, research funding is often based on promises of valorization of research findings, thus giving an extra impetus for quick marketing of applications. In order to organize and fund premarket assessment, a combined effort of stakeholders is recommended. The European Diagnostic Manufacturers Association may serve as an example of self-organization (or function as a platform) to unite the diagnostic industry and stimulate interaction with regulatory bodies in drawing new guidelines.

\section{Solidarity in the wake of personalized medicine}

In the near future, it might become increasingly possible to understand the functioning of the genome on a more individual level (including the individual as part of a subpopulation) and to stratify prevention, medication, therapies or lifestyle interventions to individual needs or the needs of their subpopulation. This will only be possible if people are to be reimbursed for a substantial part of their health-care cost. A system of collective insurance is needed to realize the potential of stratified medicine.

In this way, the danger of furthering inequalities, or unfair discrimination in health care as a consequence of increasing genetic knowledge, may be reduced.

\section{Legal aspects}

We recommend that governments proceed with additional nondiscrimination legislation in relation to genetic information in order to ensure that both employers and insurance companies use genetic information in a responsible and ethically justified manner. Fear of social and economic drawbacks should not discourage people from obtaining information about their genetic constitution for health purposes. Unfair discrimination on the basis of the constitution of one's genome should be avoided.

Research and development of new test devices are costly. Patenting may be a way to ensure return on investment; however, research may be hampered by legal restrictions on use and dissemination of genetic knowledge. A concerted action between stakeholders in industry, health care, professional and patient organizations, and governmental bodies is necessary to balance the interests of the industry with the requirements of furthering the availability of testing devices in a responsible manner.

Storage of tissue and information on the health and lifestyle of individuals in biobanks may be useful for research. Legislation is necessary to secure privacy and non-discrimination regarding genetic information to ensure an enduring public trust.

European member states should sign and ratify the European Convention on Human Rights and Biomedicine (http://conventions.coe.int/Treaty/EN/Treaties/html/164.htm).

\section{Ethical and social aspects}

The information that becomes available through tests with a low predictive value differs in several respects from genetic testing for monogenic disorders, including monogenic subsets of common complex disorders. As a genetic susceptibility test usually only reveals a slightly increased (or slightly decreased) risk of developing a certain disorder, the psychological impact, as well as the societal consequences for insurers and employers, might be less evident. There may, however, be circumstances in which poorly predictive tests give rise to psychological harm, for example, attempts to predict psychiatric disorders in children. More research is necessary to understand the ways in which people will respond to receiving this kind of risk information, as well as the social and ethical consequences of susceptibility testing and screening.

\section{Role for clinical geneticists in health care}

As genetic knowledge will become more important in managing various disorders, the specialist knowledge of clinical genetics should be made available and should be easily accessible for other professionals in health-care settings. To prepare health care for genetic testing in common disorders, cooperation between geneticists and other professionals in health care is paramount. 
Initiatives to form multidisciplinary teams, genetic resource centers, knowledge transfer centers, genetic knowledge parks, etc, should be encouraged.

Counselling in relation to testing for common complex disorders As testing for variants with a low predictive value differs from testing for monogenic disorders, the need for counselling may differ for different types of disorders and the established risk.

If the test is or is claimed to be capable of detecting high relative risks for serious conditions and thus has implications for treatment or prevention in the person or his or her near relatives, then pre- and post-test genetic counselling is needed. At present, this is rarely the case in common complex diseases when testing healthy individuals with a non-contributory family history. However, should the need for counselling arise in certain cases, the experience of clinical geneticists can be valuable to support or educate other health-care providers. Clinical geneticists should try to avoid confusion between tests with high vs low predictive value by contributing to the education and information of public and professionals.

\section{Training}

Genetic literacy of health-care professionals should be considerably improved to enable them to assess whether genetic testing, including tests with a low predictive value, may be of use, as well as to respond to the questions from patients regarding genetic testing or the information obtained from commercial genetic testing.

\section{The public}

High-quality information on genetic testing for common complex disorders should be readily available to the public at large. One of the means to achieve this would be using national and supranational trusted websites offering independent high-quality information on genetic tests, including tests with a low predictive value, and the benefits vs drawbacks of genetic testing. A concerted effort is necessary in secondary education and public education to stimulate genetic literacy.

Patient organizations may have an intermediary role in informing the public. Written documentation (leaflets, brochures) based on expertise from patient organizations and genetic specialists may be used by patients to inform primary-care professionals who often lack sufficient knowledge to detect or manage disorders with a genetic component.

\section{Developing countries}

For developing countries, the first priority in genetics must be to offer diagnosis of, and care for children and adults with inherited disabilities associated with monogenic, high penetrant inheritance, followed by action for the prevention of related morbidity and mortality. Applications of genetic testing for common complex disorders have mainly been developed for health care in wealthy countries. For the prevention and control of common complex disorders in the poorest countries, much is to be gained by raising standards of living conditions (nutrition, avoidance of deleterious environmental agents) and access to primary health care. In countries where infectious diseases and nutritional problems start to have a smaller impact, genetic testing for common complex disorders may become relevant for reducing the impact of common diseases. Genetic research in pathogenic organisms may need prioritization here. Research should focus on the specific genetic structure and health needs of populations. Health-care priorities of developing countries should be reflected in global research priorities. Improving the ability of governments for regulation and oversight of new genetic technologies will do much to bring rationality and evidence base to genetic testing and counter the 'genohype' that is affecting developed countries today. If the clinical utility and cost efficiency of a genetic test is proven, governments of developing countries have the extra duty of avoiding an access gap between those who can and those who cannot pay.

\section{RECOMMENDATIONS}

(1) Human genetics and genomics researchers should avoid generating too high expectations of the applications of their results for diagnosis, treatment and prevention.

(2) Evaluation of the clinical utility of genetic testing possibilities for common complex disorders should take place before large-scale applications.

(3) Studies in which the potential for translation of research findings to the clinic is investigated are urgently needed. Prioritization should follow generally accepted quality criteria for good health care. Tests of proven clinical utility and cost effectiveness should be implemented first.

(4) Where clinical utility is likely, but evidence is partly lacking, studies need to accompany the implementation in pilot programmes.

(5) Monogenic conditions can serve as examples for common complex diseases, both in strategies to identify etiological pathways and in strategies to develop health care in a responsible way.

(6) Sufficiently qualified health-care professionals should be available when genetic tests for common disorders are offered directly to the consumer. These professionals should be able to interpret genetic and other risk information and provide genetic counselling where applicable.

(7) Adequate regulation is necessary to guarantee truth-in-labelling and truthful promotion of genetic tests as in vitro diagnostic devices. The IVD Directive could be adapted to accommodate this. Both pre-market review and post-marketing evaluation are needed.

(8) Genetics in common disorders may lead to tailoring of health care to the needs of individuals or subpopulations. Stratified medicine will only be successful if health-care insurance is based on solidarity.

(9) Especially in developing countries, governments have an extra duty to avoid an access gap to genetic testing with proven clinical validity and cost-efficiency.

(10) European member states should sign and ratify the European Convention on Human Rights and Biomedicine (http://conventions.coe.int/Treaty/EN/Treaties/html/164.htm) and secure privacy and non-discrimination regarding genetic information.

\section{CONFLICT OF INTEREST}

The authors declare no conflict of interest. 